\title{
Use of damping identification technique for damage detection
}

\author{
Vikas Arora* \\ Department of Technology and Innovation, University of Southern Denmark, 5230 Odense, Denmark
}

\begin{abstract}
Stiffness-based structural health monitoring methods are widely used for detecting the damage in a structure. These stiffness-based structural health monitoring methods uses change in natural frequencies and modeshapes for damage detection. These methods are based on identifying the change in stiffness of the healthy and damage structure to predict the damage in the structure. These stiffness-based methods are not efficient for detecting a small damage in a structure as there is a negligible change in natural frequencies and modeshapes due to a small damage in a structure, however the damping characteristics of the structure are highly sensitive to the damage in a structure. In this paper, new damping-based damage detection procedure has been proposed. In the proposed procedure, the changes in damping matrix of the structure has been used to detect the damage in the structure. The proposed procedure is able (or can) to detect both the location of the damage and the extend of the damage in the structure. The proposed procedure of dampingbased damage detection is a 2 -step procedure. In the first step, damping matrices of both the healthy and damage structure are identified and in the second step, the identified damping matrices are used for damage detection. Numerical and experimental case studies are presented to demonstrate the effectiveness of the proposed procedure. The results have shown that the proposed damping-based damage detection procedure can be used for detecting damage in a structure with confidence.
\end{abstract}

\section{Introduction}

Stiffness-based methods have been developed and applied for detecting the damage in the structures. The basic idea behind these methods is that modal properties (natural frequencies and modeshapes) of the structure depends upon the stiffness of the structure. Therefore, the variation in the stiffness of the structure cause changes in the natural frequencies and modeshapes of the structure. Damage in a structure may be due to cracks, loose bolts, broken welds, corrosion and fatigue. Cawley and Adams [1] and Kim et al. [2] used natural frequencies of the structure to detect the damage in the structure. These methods are very simple as natural frequencies can be measured with high accuracy. Pandey and Biswas [3] used natural frequencies and modeshapes for detecting the damage in the structure. This method predicts changes in flexibility, which is inverse of stiffness, of the structure to detect damage. These methods are non-parametric and direct. However, these methods can't be used for predicting the exact location, extend of the damage and multiple damages in the structure.

Finite element method (FEM) is the most widely used for predicting the dynamic characteristics of any structure. It is well known that finite element predictions will be erroneous due to inevitable difficulties in modelling of joints, boundary conditions and damping. The experimental data are generally considered to be more accurate. This has led to the development of model updating which aims at reducing the inaccuracies present in the analytical model in the light of measured dynamic test data. Many model updating methods have been proposed in recent years. Model updating methods can be broadly classified into direct methods, (These methods are essentially non-iterative) and the iterative methods. A significant number of methods, (Baruch and Berman and Nagy [4]; Baruch [5]), which were first to emerge belonged to the direct category. Although, these methods are computationally cheaper and reproduce the measured modal data exactly, they violate structural connectivity and updated structural matrices are difficult to interpret. On the other hand, by using iterative methods, structural connectivity can be easily maintained and corrections suggested in the selected parameters can be physically interpreted. These methods also provide a wide choice of parameters for updating. Iterative methods either use eigendata or frequency response functions (FRF). Collins et al. [6] used the eigendata sensitivity for analytical model updating in an iterative framework and Lin and Ewins [7] used measured FRF data to update an analytical model. The finite element method along with model updating (Jaishi and Ren [8]; Wu and Li [9]) has been used to detect and locate the damage elements in a structure. Most of the model updating methods are iterative and parametric undamped methods and uses changes in the stiffness for predicting the damage in the structure. These methods can predict multiple damages in the structure however,

\footnotetext{
* Corresponding author: viar@iti.sdu.dk
} 
these methods can't be used to detect small damages in the structure due to low sensitivity of natural frequencies and the modeshapes to damage.

Some research has been carried out to detect the damage using damping instead of stiffness. Zhou and Yang [10] demonstrated that visually undetectable cracks have very little change in natural frequencies and modeshapes, while there is a significant change in damping characteristics of the structure. Kawiecki [11] studied the changes in modal damping, obtained using half-power bandwidth method, to detect damage in the structure and concluded that changes in damping characteristics of the structure can be a useful indicator of damage in the structure.

In this paper, new damping-based damage detection procedure has been proposed. In the proposed procedure, the changes in damping matrix due to damage are used for detecting the damage in a structure. The proposed procedure of damping-based damage detection is a 2step procedure. The proposed damping-based damage detection procedure not only predict the location of the damage but also the extent of damage in the structure. In the first step, damping matrices of both the healthy and damage structure are identified using finite element model updating approach and in the second step, the identified damping matrices are used for damage detection. Numerical and experimental case studies are presented to demonstrate the effectiveness of the proposed procedure. The results have shown that the proposed damping-based damage detection procedure can be used for detecting damage in a structure with confidence.

\section{Simulated numerical case study}

In this section, a simulated numerical case study of damped lumped mass system is presented to demonstrate that the changes in damping is a better indicator of damage in a dynamic system in comparison to stiffness. 4 degree of freedom lumped mass system shown in the Fig. 1. In the first case, the system is considered undamped and stiffness of one spring $\left(\mathrm{K}_{4}\right)$ is varied to demonstrate the damage in the system. In the second case, spring stiffnesses are considered constant and damping $\left(\mathrm{C}_{4}\right)$ is varied to simulate damage in the system. The lumped masses $\mathrm{M}_{1}, \mathrm{M}_{2}, \mathrm{M}_{3}, \mathrm{M}_{4}$ have a constant value of $5 \mathrm{Kg}$ each. In the first case, stiffness of springs $\mathrm{K}_{1}, \mathrm{~K}_{2}$ and $\mathrm{K}_{3}$ are $2 \times 10^{6} \mathrm{~N} / \mathrm{m}$ each. For simulated healthy structure, stiffness of spring $K_{4}$ is $2 \times 10^{6} \mathrm{~N} / \mathrm{m}$ considered. To simulate damage in the system, stiffness of spring 4 $\left(\mathrm{K}_{4}\right)$ is decreased by 10 to $50 \%$. The natural frequencies are calculated for different level of damage in the system. The percentage change in natural frequencies at various modes due to percentage change in stiffness value of $\mathrm{K}_{4}$ is shown in Fig. 2. It can be observed from Fig. 2 that there is maximum change of $15.53 \%$ in natural frequency with respect to $50 \%$ change in stiffness value of $\mathrm{K}_{4}$ for second mode.

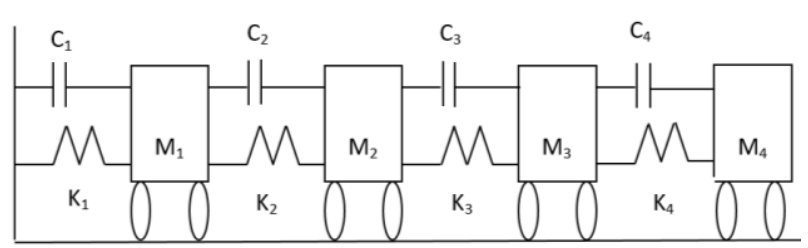

Fig. 1. Lumped mass system

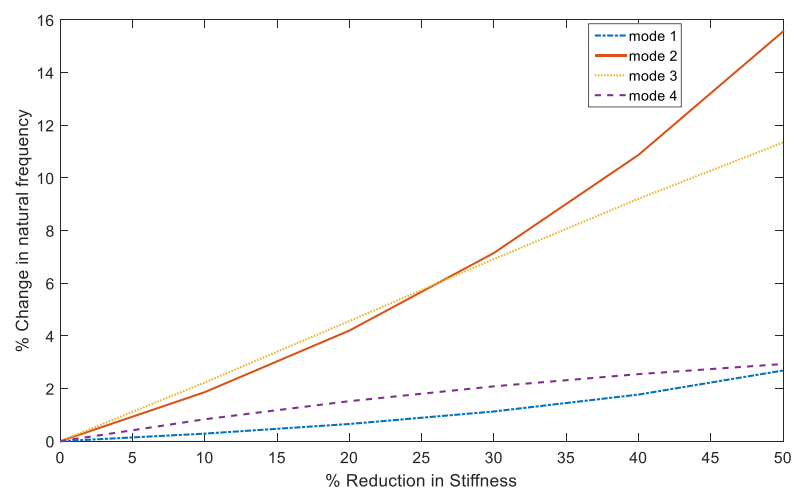

Fig. 2. \% Change in natural frequencies with change in stiffness $\left(\mathrm{K}_{4}\right)$

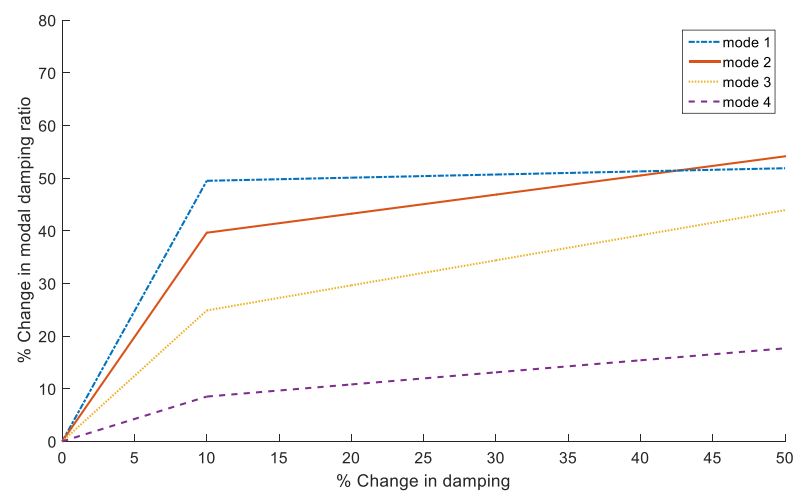

Fig. 3. \% Change in modal damping values with change in damping

A similar study has been carried out in which the damage is represented by change in damping. In this case, lumped masses $\mathrm{M}_{1}, \mathrm{M}_{2}, \mathrm{M}_{3}$ and $\mathrm{M}_{4}$ have a constant value of $5 \mathrm{Kg}$ each and stiffness of springs $\mathrm{K}_{1}, \mathrm{~K}_{2}, \mathrm{~K}_{3}$ and $K_{4}$ have constant values of $2 \times 10^{6} \mathrm{~N} / \mathrm{m}$ each. Viscous damping values for $\mathrm{C}_{1}$ is $400 \mathrm{Ns} / \mathrm{m}, \mathrm{C}_{2}$ and $\mathrm{C}_{3}$ are 300 $\mathrm{Ns} / \mathrm{m}$ each. For simulated healthy structure, $\mathrm{C}_{4}$ is 400 $\mathrm{Ns} / \mathrm{m}$. The damping value increase with the damage, therefore to simulate damage, the value of damping $\left(\mathrm{C}_{4}\right)$ has been increased by 10 to $50 \%$. The change in modal damping values due to change in damping has been calculated. The modal damping is calculated using the formulae given below.

$$
\zeta_{i}=\frac{-\alpha_{i}}{\sqrt{\alpha_{i}^{2}+\beta_{i}^{2}}}
$$

The desired damping matrix is also provided to create a better understanding of the process. Because this is a 
contrived example, the described result is known ahead of time. The percentage change in modal damping values due to change in damping values $\mathrm{C}_{4}$ is shown in Fig. 3. It can be observed from the Fig. 3 that maximum change in second modal damping due to $50 \%$ change in damping value. Fig 4. shows change in natural frequency and modal damping for the first mode with respect to change in stiffness and damping values. It can be observed from the Fig. 4 that modal damping is very sensitive to change in damping as compare to the natural frequency with respect to change in stiffness.

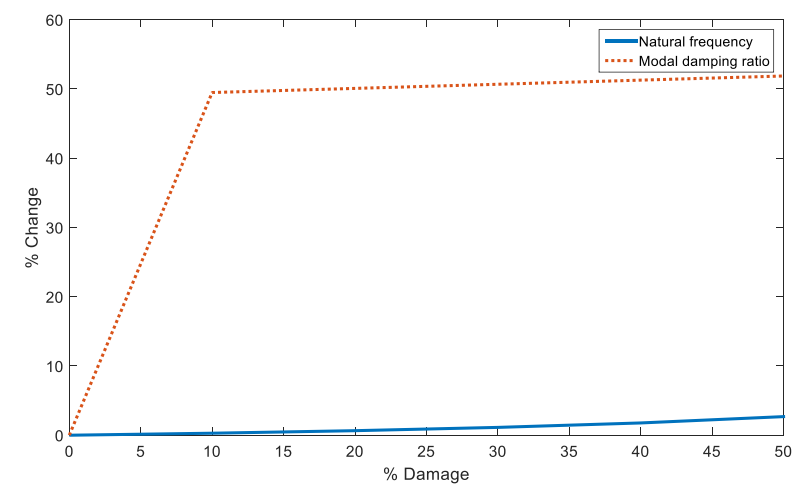

Fig. 4. Change in modal damping and natural frequencies for first mode with respect to damage.

It can be concluded from this simulated experimental study that modal damping is very sensitive with respect to damage and therefore can be used for detecting damage in the structure.

\section{Theory}

In this section, damping identification method [12] is presented. The damping identification method, this damping identification method requires prior knowledge of accurate stiffness and mass matrices. The identified damping matrix (C) is both symmetric and positive definite. This method is based on Lancaster's formulation [13] in which damping matrix is identified from the measured complex modal eigen data and damping matrix can be computed from the formula given as:

$$
C=-M\left(\Phi \Lambda^{2} \Phi^{T}+\overline{\Phi \Lambda^{2} \Phi^{T}}\right) M
$$

where the overall bar represents complex conjugate. $\Lambda$ is the diagonal matrix of complex eigenvalues $\lambda_{i}$ and $\Phi$ are complex eigenvectors. This formulation requires normalized complex eigenvectors ( $\Phi$ ). The complex eigenvectors are normalized by direct method given by Arora et al. [12] as:

$$
\Phi_{i}^{T}\left(M \lambda_{i}^{2}-K\right) \Phi_{i}=\lambda_{i}
$$

where $\mathrm{M}$ and $\mathrm{K}$ are accurate mass and stiffness matrices. The accurate mass and stiffness matrices can be obtained by undamped finite model updating method [14].

\section{Experimental case study}

An experimental study on an aluminium cantilever beam is also conducted to evaluate the effectiveness of the proposed method. The dimensions of the beam are $600 \times 50 \times 20 \mathrm{~mm}$ as shown in Fig. 5. To evaluate the proposed damping based damage detection method, a small damage has been induced in the cantilever structure as shown in Fig 6. The structure is excited by an impact hammer and responses are measured by accelerometer. The structure is excited at 5 different locations, so 5 frequency response functions (FRFs) are acquired both for healthy and damaged structures.

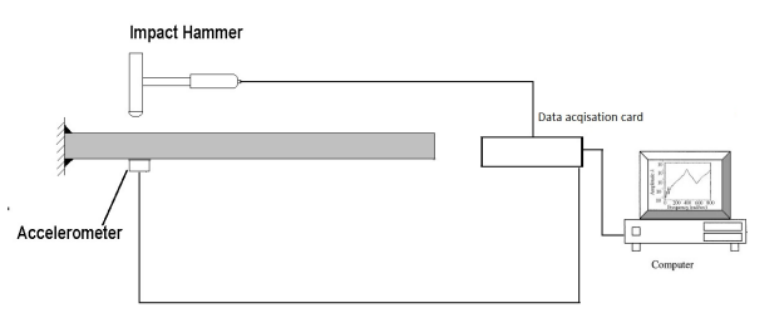

Fig. 5. Experimental setup for modal testing of cantilever beam.

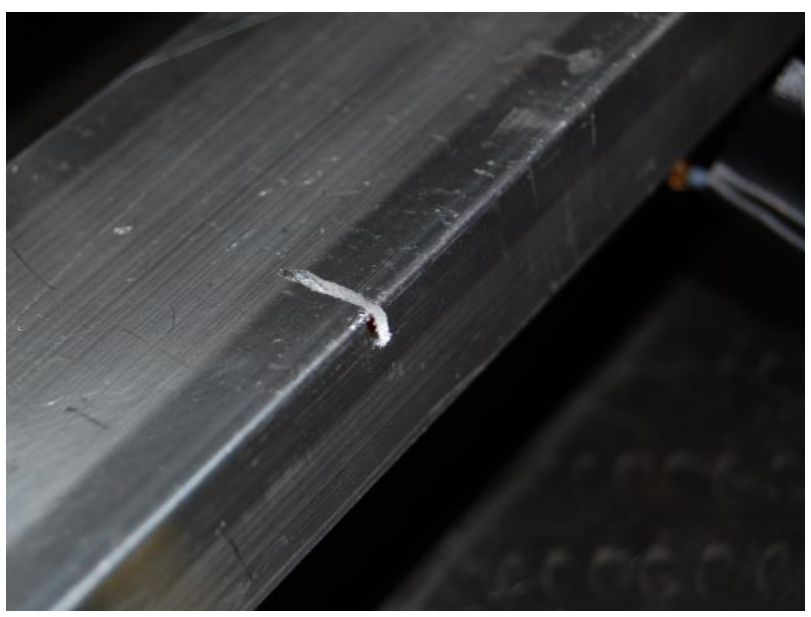

Fig. 6. Pictorial view of induced damage in the cantilever beam

The FRFs of the healthy and damaged cantilever beam are plotted in Fig.7. It can be observed from the Fig. 7 that there is no change in natural frequencies due to damage in the cantilever beam. It can be concluded from the healthy and damaged FRFs that existing stiffnessbased damage detection methods cannot be applied to detect small damage. The proposed damping-based damage detection method has been applied to detect both location and extend of the damage in the cantilever beam structure. 


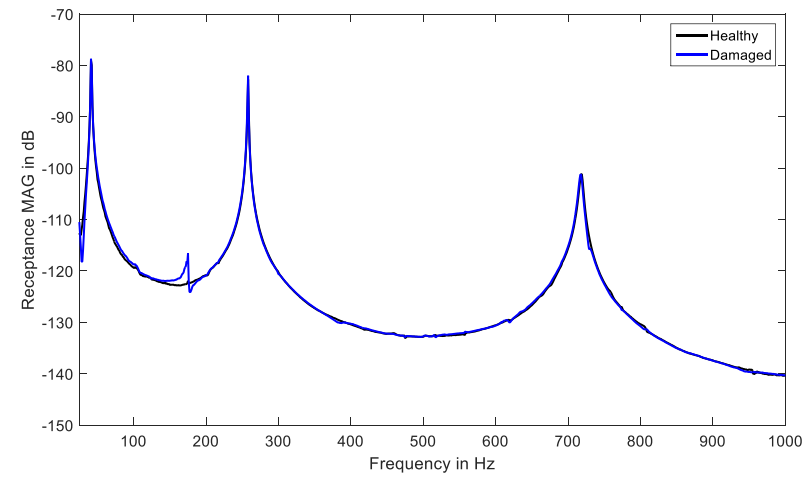

Fig. 7. Overlay of FRFs obtained from healthy and damaged cantilever beam structure.

The beam is modelled using five, two-dimensional frame elements (Two translational degrees of freedom in $\mathrm{x}$ and $\mathrm{y}$ direction and one rotational degree of freedom) and the fixed end is modelled by taking coincident nodes. Thus, two nodes that are geometrically coincident are taken as fixed end instead of one node. A horizontal, a vertical and a torsional spring couples two nodes at each of such coincident pair of nodes and the stiffness of these springs is $\mathrm{K}_{\mathrm{x}}, \mathrm{K}_{\mathrm{y}}$ and $\mathrm{K}_{\mathrm{t}}$ respectively. An overlay of the measured FRF from healthy cantilever beam and the corresponding undamped FE model FRF is shown in Fig. 8. It can be observed from Fig. 8 that the FRF predicted by FE model doesn't match with the corresponding experimental FRF, which is due error in the stiffness matrix of the FE model. For better estimation of damping, the stiffnesses at the end of the cantilever beam are updated and subsequently damping is identified using mass and updated (accurate) stiffness matrices.

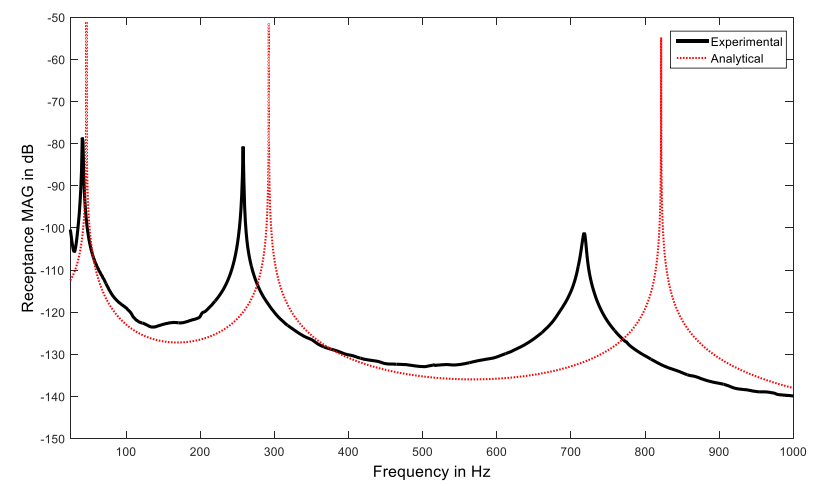

Fig. 8. Overlay of experimental FRF of the healthy structure and analytical FRF.

Undamped FE model is updated using resonance and anti-resonance frequencies [14]. Choice of updating parameters based on engineering judgment about the possible locations of modelling errors in a structure is one of the strategies to ensure that only physical meaningful corrections are made. In case of cantilever beam structure, modelling of stiffness at the end is expected to be the dominant source of inaccuracy in the FE model. The three spring stiffnesses at the end are chosen as updating variables. Natural frequencies and anti-resonance frequencies in the range of $0-1000 \mathrm{~Hz}$ are selected for updating the finite element model. The initial and final values of three springs at the end are given in the Table 1. It is observed that the values of stiffness of all the springs at the end are reduced. Fig. 8 shows the overlay of measured healthy and undamped updated FRF. It can be observed from Fig. 9 that the shape of the updated FRFs is same as that of measured FRFs. Since the undamped updating of FE model is performed, experimental FRF and updated FRF do not match near resonance and anti-resonance frequency points as the effect of damping is maximum near the resonance and anti-resonance regions.

Table 1. Stiffness values of springs before and after updating.

\begin{tabular}{|c|c|c|}
\hline $\begin{array}{c}\text { Updating } \\
\text { variable }\end{array}$ & Initial Value & Updated Value \\
\hline $\begin{array}{c}\mathrm{K}_{\mathrm{x}} \\
(\mathrm{N} / \mathrm{m})\end{array}$ & $3.28 \times 10^{6}$ & $1.41 \times 10^{5}$ \\
\hline $\begin{array}{c}\mathrm{K}_{\mathrm{y}} \\
(\mathrm{N} / \mathrm{m})\end{array}$ & $3.28 \times 10^{6}$ & $1.53 \times 10^{5}$ \\
\hline $\begin{array}{c}\mathrm{K}_{\mathrm{t}} \\
(\mathrm{N} \mathrm{m} / \mathrm{rad})\end{array}$ & $3.28 \times 10^{6}$ & $1.89 \times 10^{5}$ \\
\hline
\end{tabular}

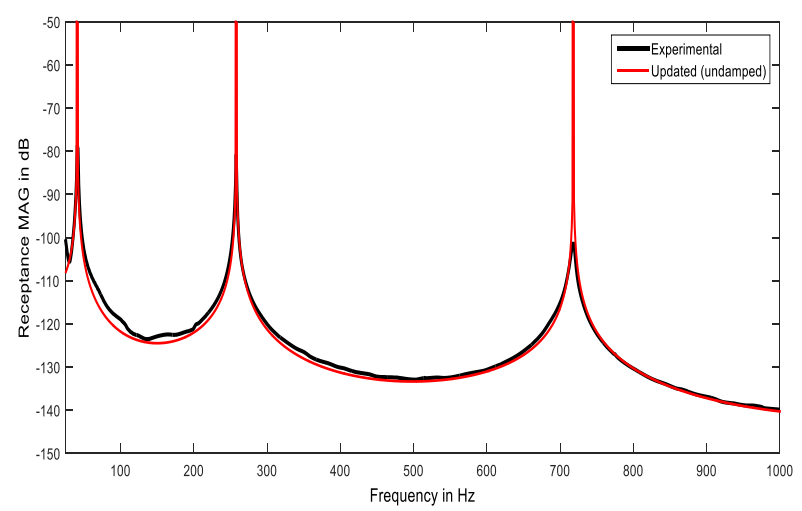

Fig. 9. Overlay of experimental FRF of the healthy structure and updated FRF

The updated FE model obtained in the previous step is used to identify the viscous damping matrix of the cantilever beam structure. The size of complex eigendata matrices is $[5 \times 5]$ whereas, the size of mass and stiffness matrices obtained in previous step is $[18 \times 18]$. To apply the damping identification method, the size of mass and stiffness matrices are reduced to [5×5] according to the measured degrees of freedom using iterated IRS method [15] given by Friswell et al. Damping of the healthy cantilever beam is identified using damping identification method using accurate mass and stiffness matrices. Fig. 10 shows the overlay of experimental and corresponding damped identified FRF for healthy cantilever beam structure. It can be observed from Fig. 10 that the 
damping identification method is able to predict FRF accurately up to considered frequency range $0-1000 \mathrm{~Hz}$. Similarly, same procedure is applied to identify the damping matrix for the damage cantilever beam structure.

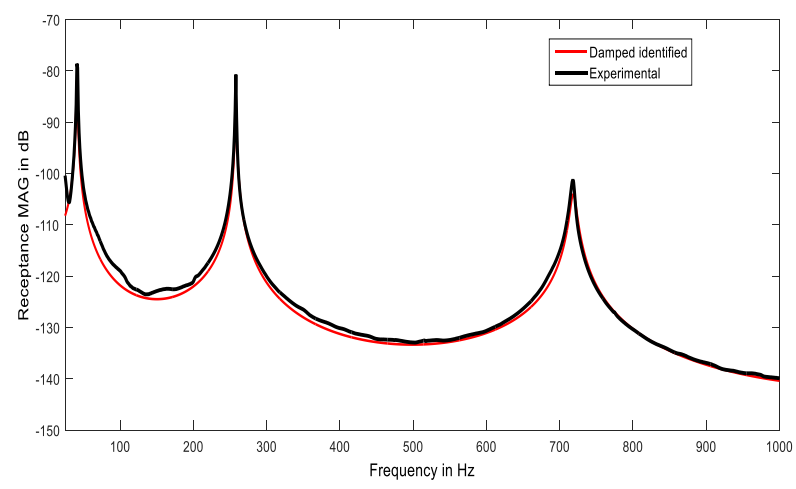

Fig. 10. Overlay of experimental FRF of the healthy structure and damped updated FRF

It can be observed from the identified damping matrices of the healthy and damaged cantilever beam that there is a $3.06 \%$ damage in the element 3 of the cantilever beam structure. It can be concluded from the experimental study that the proposed damping-based damage detection method is able to predict small damage in a structure.

\section{Conclusions}

In this paper, a damping-based damage detection method method is proposed. The proposed method can detect the location as well as the extent of the damage in a structure. The proposed damping-based damage detection method can detect a small damage in a structure as modal damping is very sensitive to small changes in the damping matrix due damage in the structure. Whereas, stiffness-based damage detection cannot able to detect small damages in the structure as natural frequencies and modeshapes are not very sensitive to small changes in stiffness matrix due to damage. Numerical and experimental case studies based on a lumped mass system and cantilever beam structure have been carried out to demonstrate that the changes in modal damping is better indicator of damage in a structure than the natural frequencies and modeshapes. The proposed method is subsequently applied to real experimental data of the cantilever beam structure to detect small damage in the structure. The proposed method able to detect successfully small damage in the cantilever beam structure. So, it can be concluded that damping-based damage detection method can be applied with confidence.

\section{References}

1. P. Cawley, R. Adams, J. Strain Anal. Eng. 14, (1979)

2. J. T. Kim, H.M. Cho, N. Stubbs, Eng. Struct. 25 (2003)
3. A.K. Pandey, M. Biswas, J. Sound Vib. 169 (1994)

4. A. Berman, E.J. Nagy, AIAA J. 21 (1983)

5. M. Baruch, AIAA J. 22 (1984)

6. J.D. Collins, G.C. Hart, T.K. Hasselman, B. Kennedy, AIAA J. 12 (1974)

7. R.M. Lin, D.J. Ewins, Mech. Syst. Sig. Pro. 8 (1994)

8. B. Jaishi, W.X. Ren, J. Sound Vib. 290 (2006)

9. J.R. Wu, Q.S. Li, J. Constr. Steel Res. 62 (2006)

10. L. Zhou and G. Yang, Smart Struct. Syst. 2 (2006)

11. G. Kawiecki, European COST F3 conference on system identification and structural health monitoring (Madrid, Spain, 2000)

12. V. Arora, S.P. Singh, T.K. Kundra, J. Sound Vib. 324 (2009)

13. P. Lancaster, J. Aero. Sc. 28 (1961)

14. V. Arora, Inv. Prob. Sc. Eng. 22 (2014)

15. M.I. Friswell, S.D. Garvey, J.E.T. Penny, J. Sound Vib. 1211 (1998) 